\title{
Electron population during the cascade of kaonic nitrogen atoms
}

\author{
T. Koike \\ Advanced Meson Science Laboratory, RIKEN, \\ Wako-shi, Saitama 351-0198, Japan
}

\begin{abstract}
The cascade calculation of kaonic nitrogen atoms is performed and the electron population during the cascade is investigated to be useful for the future kaon mass measurement at DA $\Phi$ NE. It is found that the one electron remains in the K-shell with the probability of $4 \%$ at the the moment of the $6 \rightarrow 5$ kaonic $\mathrm{x}$-ray emission in the gaseous target at a density $\rho=3.4 \rho_{\mathrm{NTP}}$.
\end{abstract}

\section{Introduction}

The Particle Data Group assigned $493.677 \pm 0.013 \mathrm{MeV}$ to the charged kaon mass as a world average[1]. However, there exists a serious disagreement between the most two recent mass measurements using kaonic atom x-rays, in which the deduced masses differ about $60 \mathrm{keV}$ although their uncertainties are about $10 \mathrm{keV}$. In order to settle this discrepancy, new precise charged kaon mass measurement using kaonic nitrogen atom x-rays in a gaseous target is planned at the $\mathrm{DA} \Phi \mathrm{NE}[2,3]$.

For the precise kaon mass determination, there is another problem on the theoretical side. The electron screening effect on the kaonic x-ray energy is difficult to estimate correctly because it needs the knowledge of the electron population at the moment of $\mathrm{x}-$ ray emission, which depends on the balance between Auger electron emission and electron refilling during the atomic cascade process. The incorrect estimation of electron screening effect might cause several $10 \mathrm{keV}$ errors in kaon mass for the worst case.

Our purpose is to develop the cascade code for kaonic nitrogen atoms, which enables us to determine the electron fraction at each kaon atomic level during the cascade. Our previous cascade model employed in Ref.[2] is improved to distinguish 2s- and 2p-orbit in the electron L-shell and new results are given in this paper. 


\section{Cascade model}

The cascade calculation starts from kaon principal quantum number $n_{\text {init. }}=30$. For kaon sector, we include

- E1 radiative transition

- internal Auger process for ejecting 1s-, 2s- and 2p-electron

- nuclear absorption by strong interaction

- weak decay

and for electron sector,

- $2 \mathrm{p} \rightarrow 1$ s radiative transition

- KLL-Auger process between two 2s-electrons, one $2 \mathrm{~s}$ - and one 2p-electron, and two 2p-electrons

- electron pick-up from outside to L-shell

are taken into account. These rates are shown in Fig.1 and Table.1.

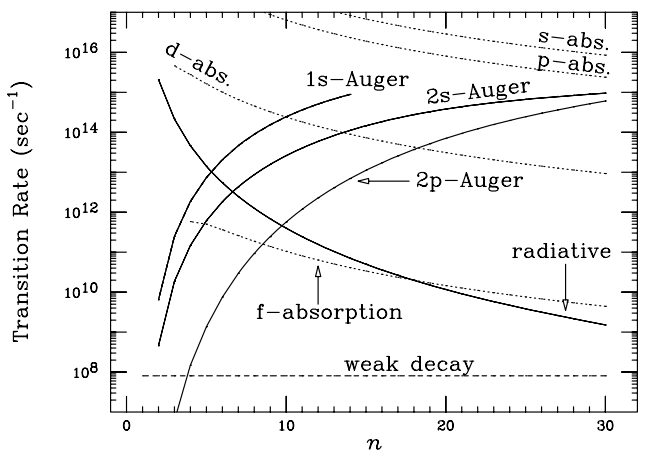

Table 1: The rates for electron refilling processes.

\begin{tabular}{cr}
\hline$\Gamma_{2 \mathrm{p} \rightarrow 1 \mathrm{~s}}^{\mathrm{rad}}$ & $0.8 \times 10^{12} \mathrm{~s}^{-1}$ \\
$\left.\Gamma_{(2 \mathrm{sL}}^{\mathrm{KLL}}\right)$ & $38.2 \times 10^{12} \mathrm{~s}^{-1}$ \\
$\Gamma_{(2 \mathrm{~s}, 2 \mathrm{p})}^{\mathrm{KLL}}$ & $10.2 \times 10^{12} \mathrm{~s}^{-1}$ \\
$\Gamma_{(2 \mathrm{pL}, 2 \mathrm{p})}^{\mathrm{KL}}$ & $9.7 \times 10^{12} \mathrm{~s}^{-1}$ \\
$\Gamma^{\text {pick }}$ & free parameter \\
\hline
\end{tabular}

Figure 1: The rates for various processes of kaon as a function of $n$. As for the radiative and Auger processes, only the rates for circular orbit $(n=l-1)$ are shown.

In our previous model employed in Ref.[2], the electron transfer process from L- to Kshell is described by using one free parameter. In the present model, on the other hand, the radiative and the KLL-Auger process are explicitly included and no free parameter is used for $\mathrm{L} \rightarrow \mathrm{K}$ electron transfer process. The electron pick-up rate from the surrounding atoms to L-shell, $\Gamma^{\text {pick }}$, is expected to be the order of $10^{10} \mathrm{~s}^{-1}$ for the thermalized atom, but it is left as a free parameter because the velocity distribution of exotic atoms during the cascade is unknown. 
The initial kaon angular momentum distribution is assumed to be $P(l) \propto(2 l+1) \exp (a l)$ where $a$ is a free parameter. As for the initial electron populations, several electrons can be lost at the formation of kaonic nitrogen atoms involving the break-up process of nitrogen molecule. For simplicity, the 1s-orbit is assumed to be filled at start, but 2s- and 2 p-electron populations are left as a free-parameters,

\section{Fitting to x-ray yields}

The free-parameters in our present cascade model are adjusted to reproduce the experimental x-ray yields observed at DA $\Phi N E[2]$. The best parameters in the $\chi^{2}$-fit are listed in Table II and the calculated x-ray yields are shown in Table III. It should be noted that the $8 \rightarrow 6$ x-ray energy $(\sim 7.54 \mathrm{keV})$ is very close to $6 \rightarrow 5$ one $(\sim 7.58 \mathrm{keV})$ and these are not observed as the distinct peaks in the present experimental resolution. Therefore, the fitting to the $\mathrm{x}$-ray yields has been done by assuming that the experimental ' $6 \rightarrow 5$ ' yield is the sum of the $6 \rightarrow 5$ and the $8 \rightarrow 6$ yields. It is also noted that we can determine only

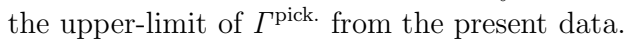

Table 2: Best-fitted cascade parameters in the $\chi^{2}$-fit to the x-ray yields.

\begin{tabular}{cc}
\hline \hline \multicolumn{2}{c}{ kaon initial distribution } \\
\hline$n_{\text {start }}$ & $30-$ fixed \\
$a[$ in $\exp (a \ell)]$ & 0.0 \\
\hline \hline electron initial distribution ${ }^{*)}$ \\
\hline$\left(f_{1 \mathrm{~s}(1)}^{0}, f_{1 \mathrm{~s}(2)}^{0}\right)$ & $(1.0,1.0)-$ fixed \\
$\left(f_{2 \mathrm{~s}(1)}^{0}, f_{2 \mathrm{~s}(2)}^{0}\right)$ & $(0.6,0.0)$ \\
$\left(f_{2 \mathrm{p}(1)}^{0}, f_{2 \mathrm{p}(2)}^{0}\right)$ & $(1.0,0.0)$ \\
\hline \hline & electron pick-up rate \\
\hline$\Gamma^{\text {pick }} \leq 2 \times 10^{10} \mathrm{~s}^{-1}$ \\
- not well-determined \\
\hline
\end{tabular}

*) $f_{\alpha(i)}^{0}(\alpha=1 \mathrm{~s}, 2 \mathrm{~s}, 2 \mathrm{p} ; i=1,2)$ denotes the $i$-th electron population in the orbit $\alpha$.
Table 3: The experimental x-ray yields (density $\rho=3.4 \rho_{\mathrm{NTP}}$ ) and the calculated ones using the best parameter set in Table2.

\begin{tabular}{ccc}
\hline \hline$n_{i} \rightarrow n_{f}$ & cal. & exp. \pm (stat.) \pm (sys.) \\
\hline $4 \rightarrow 3$ & $62.2 \%$ & \\
$5 \rightarrow 4$ & $58.0 \%$ & $57.4 \pm 15.2 \pm 5.7 \%$ \\
$6 \rightarrow 5$ & $\left.55.0 \%^{*}\right)$ & $55.0 \pm 3.9 \pm 5.5 \%$ \\
$7 \rightarrow 6$ & $41.2 \%$ & $41.5 \pm 7.4 \pm 4.1 \%$ \\
$8 \rightarrow 7$ & $21.7 \%$ & \\
$9 \rightarrow 8$ & $7.4 \%$ & \\
$10 \rightarrow 9$ & $2.0 \%$ & \\
\hline
\end{tabular}

*) $2.4 \%$ of $8 \rightarrow 6$ yield is included.

\section{Electron population in a gaseous target}

Fig. 2 shows the averaged electron number for each electron orbit during the cascade, as a function of kaon principal quantum number. At $n \geq 20$, the 1s-electron population is constant because the K-shell Auger transition is energetically forbidden. When the $\mathrm{K}$-shell Auger process is allowed, the K-shell population rapidly falls off by successive electron emission before the electron refilling is completed. It is found that the 1s-electron 
population becomes minimum around the x-ray observed region, and the averaged 1selectron number at $n=6$ is estimated to be 0.04 .

Fig. 3 shows the details of 1s-electron configuration during the cascade. The probability that two electrons remain in the K-shell becomes almost zero at $n<10$. Thus, it is concluded that one electron remains in the 1 s-orbit with the probability of $4 \%$ at $n=6$.

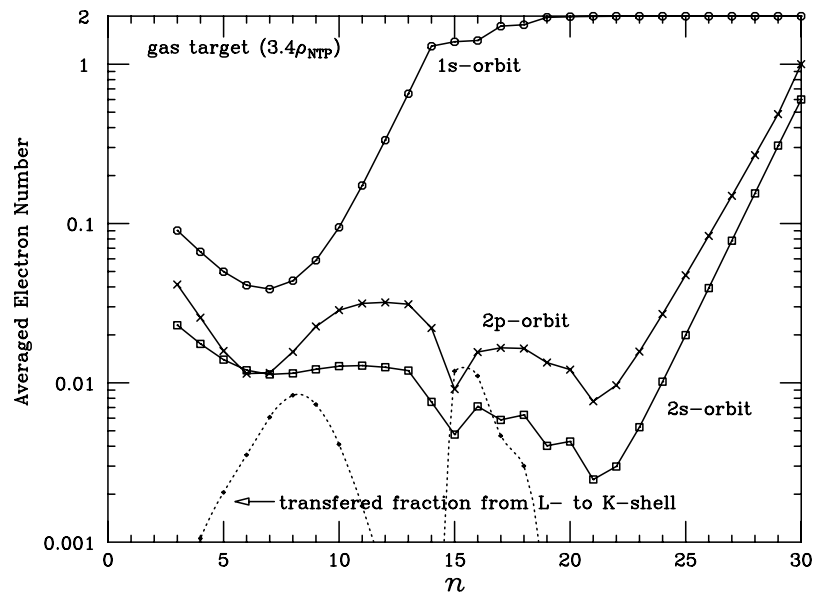

Figure 2: Average electron number during the cascade in the gaseous target at a density $\rho=3.4 \rho_{\mathrm{NTP}}$, as a function of kaon principal quantum number $n$. The dotted line denotes the transferred electron fraction from L- to K-shell.

\section{Conclusion}

As the results of the cascade calculation for the kaonic nitrogen atoms in the gaseous target at a density $\rho=3.4 \rho_{\mathrm{NTP}}$, it is found that the one 1s-orbit electron remains with the probability of $4 \%$ at the the moment of the $6 \rightarrow 5 \mathrm{x}$-ray emission. This result will be helpful for the future kaon mass measurement using kaonic nitrogen atom x-rays.

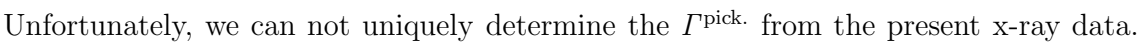
Since $\Gamma^{\text {pick. }}$ should be proportional to the target density, we can predict the density dependence of the results if $\Gamma^{\text {pick. }}$ is quantitatively determined. The $\Gamma^{\text {pick. is also related to the }}$ occurrence of "Coulomb explosion" at a formation stage[4]. Therefore, the density dependence of kaonic nitrogen atom x-ray yields is the important subject to be studied for the better understanding of the cascade mechanism, apart from the kaon mass measurement. 


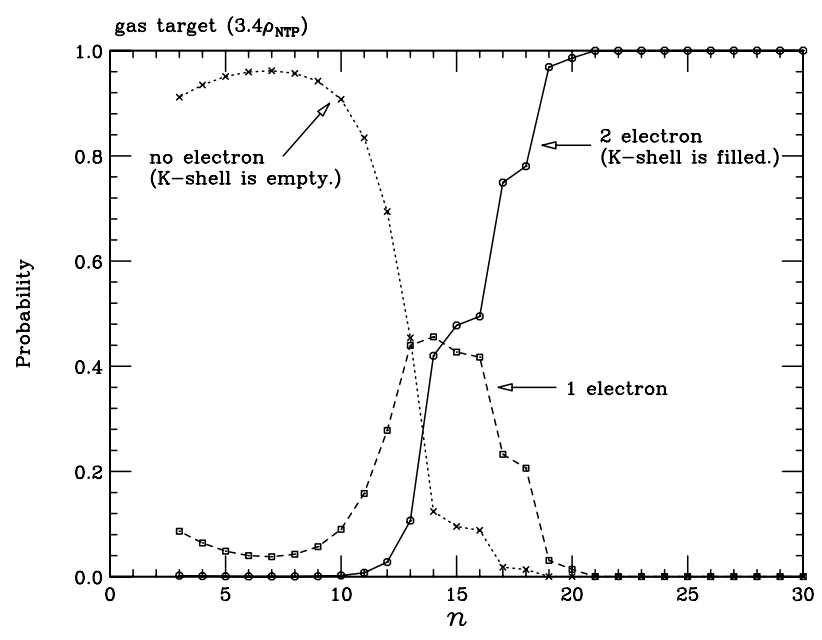

Figure 3: Electron configuration in the K-shell during the cascade in the gaseous target at a density $\rho=3.4 \rho_{\text {NTP }}$ as a function of $n$. The solid, dashed and dotted lines denote the probability that two, one and no electrons remain in the K-shell, respectively.

\section{Acknowledgments}

The author is supported by the Special Postdoctoral Researchers Program from RIKEN.

\section{References}

[1] S. Eidelman et al. (Particle Data Group), Phys. Lett. B592, 1 (2004).

[2] T. Ishiwatari et al., Phys. Lett. B593, 48 (2004).

[3] G. Beer et al., Phys. Lett. B535, 52 (2002).

[4] T. Siems et al., Phys. Lett. 84, 4573 (2000). 
